ORIGINAL ARTICLE

\title{
Effectiveness of an outside-the-boot ankle brace in reducing parachuting related ankle injuries
}

\author{
M D Schmidt, S I Sulsky, P J Amoroso
}

Injury Prevention 2005;11:163-168. doi: 10.1136/ip.2004.006304

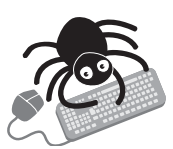

An additional table appears on our website.

See end of article for authors' affiliations .....................

Correspondence to: Dr S I Sulsky, ENVIRON International Corp, PO Box 2424, Amherst, MA 01004, USA; ssulsky@ environcorp.com

Accepted

12 December 2004
Objectives: To examine the efficacy of an outside-the-boot parachute ankle brace (PAB) in reducing risk of ankle injury to army paratrooper trainees and to identify inadvertent risks associated with PAB use.

Design: The authors compared hospitalization rates for ankle, musculoskeletal, and other traumatic injury among 223172 soldiers trained 1985-2002 in time periods defined by presence/absence of PAB use protocols. Multiple logistic regression analysis estimated adjusted odds ratios (OR) and $95 \%$ confidence intervals for injury outcomes, comparing pre and post brace periods to the brace protocol period. Setting: A research database consisting of training rosters from the US Army Airborne training facility (Fort Benning, GA) occupational, demographic, and hospitalization information.

Main outcome measures: Injuries were considered training related if they occurred during a five week period starting with first scheduled static line parachute jump and a parachuting cause of injury code appeared in the hospital record.

Results: Of 939 parachuting related hospitalizations during the defined risk period, $597(63.6 \%)$ included an ankle injury diagnosis, $198(21.1 \%)$ listed a musculoskeletal (non-ankle) injury, and 69 (7.3\%) cited injuries to multiple body parts. Risk of ankle injury hospitalization was higher during both pre-brace (adjusted $\mathrm{OR} 2.38,95 \% \mathrm{Cl} 1.92$ to 2.95 ) and post-brace (adjusted $\mathrm{OR} 1.72,95 \% \mathrm{Cl} 1.27$ to 2.32 ) periods compared with the brace protocol period. Odds of musculoskeletal (non-ankle) injury or injury to multiple body parts did not change between the brace and post-brace periods.

Conclusion: Use of a PAB during airborne training appears to reduce risk of ankle injury without increasing risk of other types of traumatic injury.
A nkle injuries are common among civilian and military parachutists. Among military paratroopers, the estimated incidence of ankle injury ranges from 1 to 4.5 per 1000 jumps, accounting for $30-60 \%$ of all parachuting related injuries. ${ }^{1-5}$ Risk of ankle injury may be higher among inexperienced parachutists, with rates between 5.4 and 7.1 per 1000 jumps. $^{67}$ As US Army paratroopers complete 200000 to 300000 static line parachute jumps each year, approximately 685 ankle injuries are expected annually. In addition to the considerable direct and indirect medical costs associated with injury, there is substantial negative impact on mission readiness. For example, an injured paratrooper may require up to four soldiers to assist in evacuation from the drop zone. ${ }^{8}$ These concerns motivated the development of an outside-the-boot parachute ankle brace (PAB).

Developed by Aircast (Summit, NJ, USA), the PAB consists of a hard plastic outer shell lined with air bladders which pad the medial and lateral malleoli, preventing extreme ankle inversion and eversion while allowing plantarflexion and dorsiflexion. In a randomized intervention trial in October 1993, the incidence of ankle sprains was reduced by $85 \%$ in PAB users undergoing training at the Army Airborne School. ${ }^{6}$ The Army subsequently adopted use of the braces for all trainees in 1994. However, the Airborne School discontinued $\mathrm{PAB}$ use seven years later due to financial concerns and anecdotal evidence of an increase in proximal injuries (knee and hip) potentially related to PAB use.

This study was conducted to determine whether PAB use reduces the occurrence of parachuting related ankle injuries among Airborne School trainees and to identify inadvertent injury risks associated with $\mathrm{PAB}$ use.

\section{METHODS}

Existing databases were used to assess the relation between $\mathrm{PAB}$ use and hospitalization for ankle injury, non-ankle traumatic injury, and multiple traumatic injuries. Subjects were identified using Airborne School student rosters, which contained data regarding each trainee's start of training date, graduation status, and reason for not graduating, if applicable. Outcome and covariate data were drawn from the Total Army Injury and Health Outcomes Database (TAIHOD), which includes biannual personnel files from the Defense Manpower Data Center (DMDC) database and hospital records from the Individual Patient Data System (IPDS). The TAIHOD has been described elsewhere. ${ }^{9}$

The three week regimen at the Airborne School calls for two weeks of land based training followed by five static line parachute jumps during the final week. The study population comprised 227549 active duty US Army personnel enrolled in the school between 1 January 1985 and 31 December 2002, including 37977 students who did not successfully complete the training course. Reasons for not graduating included failure to pass physical training, quitting, and medical and academic reasons. Non-graduates may withdraw from training either before or after the first static line parachute jump. Because data specifying the timing of withdrawal were incomplete, and injury hospitalization often results in nongraduation, all non-graduates were retained in these analyses.

Students were linked via an encrypted identification number to the closest DMDC record available within one year of the start of Airborne training. Active duty status was verified using date of entry into military service and branch of service codes. As no records were available to identify which

\footnotetext{
Abbreviations: DMDC, Defense Manpower Data Center; ICD-9-CM, International Classification of Diseases, Ninth Revision, Clinical Modification; IPDS, Individual Patient Data System; PAB, parachute ankle brace; TAIHOD, Total Army Injury and Health Outcomes Database.
} 
students participated in the 1993 Amoroso et al intervention trial, ${ }^{6}$ all students whose jump week occurred during this period were excluded $(n=1335)$. Students who completed training in the two month period following the randomized field trial and before the implementation of the PAB protocol were also excluded $(n=1852)$ due to the possibility of carryover $\mathrm{PAB}$ use. Finally, we excluded trainees with missing or unknown values for sex, race, or pay grade $(n=114)$ and those whose recorded age at entry into the Army was $<17$ or $>40$ years $(n=1076)$. Such values likely reflect data entry errors. Following these restrictions, 223172 personnel remained eligible for analysis.

Sex, race/ethnicity, age, and rank were obtained from the DMDC record. Age was categorized into approximate quintiles based on the distribution of the entire cohort, and the highest quintile group (ages 25-56) was divided into smaller units (25-29, 30-34, and 35-56 years) to better quantify the risk of injury among older students. Race/ ethnicity categories were White, Black, Hispanic, and Other. Three categories of rank were based on paygrade (junior enlisted (E1-E4), senior enlisted (E5-E9), and Commissioned and Warrant Officers).

Although there were no individual level data regarding $\mathrm{PAB}$ use, compliance with training protocols is expected to be near $100 \%$. Therefore, comparisons were made based on presence or absence of a policy requiring PAB use based on training dates. Taking into account the timing of the randomized trial and the subsequent two month transitional period, we defined brace protocol periods as outlined in table 1. Each trainee was categorized according to the protocol period that encompassed his/her third week of training ("jump week") based on their start of training date recorded in the student rosters.

Hospital records were obtained from the IPDS for all admissions between 1 January 1980 and 31 December 2002. Each record included admission and discharge dates, and up to eight diagnoses and procedures coded according to International Classification of Diseases, Ninth Revision, Clinical Modification (ICD-9-CM). Traumatic injuries were classified by Standard NATO Agreement codes of external cause, which include injuries caused by parachuting from military aircraft. Hospital records indicating both an injury diagnosis and a parachuting cause code during the five week period starting with jump week were considered training related. A five week risk period was chosen in the event that students delayed treatment until after completion of airborne training. As injury may lead to a termination of training, there is strong incentive to postpone care, if possible.

We used the Barell matrix to classify ICD-9-CM diagnoses for traumatic injuries into body regions. ${ }^{10}$ In addition, we created new categories for ankle, non-ankle musculoskeletal, and traumatic non-ankle injuries. If an orthopedic procedure code was also recorded, ankle injuries were further categorized as "severe". Students with diagnoses indicating traumatic injury to more than one distinct body part/region were classified as having multiple injuries (see http://www. injuryprevention.com/supplemental for table).

\begin{tabular}{ll} 
Table 1 & Parachute ankle brace protocol periods, 1985- \\
2002 & \\
\hline Dates & Protocol \\
\hline 1 January 1985 to 30 September 1993 & Pre-brace \\
1 October 1993 to 31 December 1993 & Excluded interval ${ }^{*}$ \\
1 January 1994 to 30 September 2000 & Brace \\
1 October 2000 to 31 December 2002 & Post-brace \\
\hline
\end{tabular}

*Includes one month intervention trial and the following two months, during which time carryover PAB use may have occurred.

\section{Statistical methods}

SAS version 8.2 was used for data management and analysis (SAS Institute, Cary, NC, USA). Descriptive characteristics for airborne trainees were calculated for each protocol period. In addition to calculating the percentage of trainees injured across protocol periods, injury rates per 1000 jumps were estimated assuming five jumps were completed per trainee, which is the requirement for graduation. As some trainees may not complete all five jumps (for example, due to hospitalization, failure of physical training), injury rates were likely underestimated.

To examine time trends, injury rates were also calculated for each study year. Annual injury rates among airborne trainees were standardized to the $1985 \mathrm{PAB}$ population distribution to adjust for age, sex, and race. Available information did not allow standardization of annual US Army injury rates. Odds ratios (OR) and 95\% confidence intervals (95\% CI) were used to quantify the relative risk of injury during the pre-brace and post-brace compared with the brace protocol period.

Separate logistic regression models were used to determine the association between each outcome and each individual covariate (age, sex, rank, race/ethnicity, history of prior injury), controlling for protocol period. Covariates associated with an injury outcome at $p \leqslant 0.20$ were entered in a preliminary multivariable model. Those with the highest $\mathrm{p}$ values were removed individually until all variables in the model were associated with the injury outcome at $p \leqslant 0.05$. Covariates that were not statistically significantly associated with injury but resulted in $\geqslant 15 \%$ change in OR for any other variable in the model were considered confounders, and retained in the final model.

\section{RESULTS}

Of the 223172 Airborne students identified, 56.7\% $(n=126603)$ entered jump week during the pre-brace period, $30.5 \%(\mathrm{n}=68140)$ during the brace period, and $12.7 \%(\mathrm{n}=28429)$ in the post-brace period. There was a higher proportion of women and older trainees during the brace period compared with other time intervals (table 2 ).

The overall rate of parachuting related injuries during the pre-brace period $(0.52 \%$ or 1.0 per 1000 jumps $)$ was twice the rate observed in the brace period $(0.26 \%$ or 0.5 per 1000 jumps) and approximately $50 \%$ higher than in the post-brace period $(0.35 \%$ or 0.7 per 1000 jumps). A total of 939 out of $223172(0.4 \%)$ students were hospitalized with a parachuting related injury within the five week risk period, $78.8 \%$ (739/939) during jump week. Almost 64\% (597/939) of all risk period injury hospitalizations were to the ankle, and $96 \%$ $(573 / 597)$ of ankle injury hospitalizations were due to fractures.

The ankle injury rate was highest during the pre-brace period ( 0.7 per 1000 jumps) compared with the brace $(0.3$ per 1000 jumps) and post-brace (0.5 per 1000 jumps) periods (table 3$)$. The majority of ankle injury hospitalizations (83\%) occurred during jump week while $11 \%$ occurred the following week. Although ankle injury hospitalization rates varied within each protocol period, annual standardized rates during the brace period were consistently lower than rates observed during non-brace periods (fig 1). When ankle injuries were restricted to those with an accompanying ankle procedure ("severe ankle injury"), the pre-brace rate decreased to $0.27 \%$ while brace and post-brace rates remained relatively stable.

Hospitalization for non-ankle traumatic injuries was also highest in the pre-brace period (table 3 ). The most common non-ankle injuries were to the head $(0.05 \%, 107 / 223172)$, foot $(0.03 \%, 63 / 223172)$, and vertebral column $(0.02 \%, 50 /$ 223 172). For each of these regions, rates were approximately 
Table 2 Descriptive characteristics of US Army Airborne School trainees, by parachute ankle brace protocol period, 1985-2002

\begin{tabular}{|c|c|c|c|c|c|c|}
\hline \multirow[b]{2}{*}{ Characteristic } & \multicolumn{2}{|c|}{ Pre-brace $^{*}(n=126603)$} & \multicolumn{2}{|c|}{ Brace protocol* $(n=68140)$} & \multicolumn{2}{|c|}{ 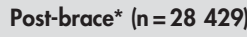 } \\
\hline & $\mathbf{n}$ & $\%$ & $\mathbf{n}$ & $\%$ & $\mathbf{n}$ & $\%$ \\
\hline \multicolumn{7}{|c|}{ Age at start of training } \\
\hline $17-18$ & 27192 & 21.48 & 10430 & 15.31 & 5549 & 19.52 \\
\hline 19-20 & 40422 & 31.93 & 20906 & 30.68 & 9093 & 31.98 \\
\hline $21-22$ & 21587 & 17.05 & 12301 & 18.05 & 4716 & 16.59 \\
\hline $23-24$ & 14338 & 11.33 & 9342 & 13.71 & 3330 & 11.71 \\
\hline $25-29$ & 16170 & 12.77 & 10529 & 15.45 & 3897 & 13.71 \\
\hline $30-34$ & 5341 & 4.22 & 3568 & 5.24 & 1458 & 5.13 \\
\hline $35-56$ & 1553 & 1.23 & 1064 & 1.56 & 386 & 1.36 \\
\hline \multicolumn{7}{|l|}{ Sex } \\
\hline Men & 120476 & 95.16 & 63237 & 92.80 & 27025 & 95.06 \\
\hline Women & 6127 & 4.84 & 4903 & 7.20 & 1404 & 4.94 \\
\hline \multicolumn{7}{|l|}{ Race/ethnicity } \\
\hline White & 96407 & 76.15 & 49352 & 72.43 & 20346 & 71.57 \\
\hline Black & 19770 & 15.62 & 9685 & 14.21 & 3378 & 11.88 \\
\hline Hispanic & 5361 & 4.23 & 5601 & 8.22 & 3282 & 11.54 \\
\hline Other & 5065 & 4.00 & 3502 & 5.14 & 1423 & 5.00 \\
\hline \multicolumn{7}{|l|}{ Rank } \\
\hline $\begin{array}{l}\text { Junior enlisted } \\
\text { (E1-E4) }\end{array}$ & 102449 & 80.92 & 54337 & 79.74 & 24098 & 84.77 \\
\hline $\begin{array}{l}\text { Senior enlisted } \\
\text { (E5-E9) }\end{array}$ & 11554 & 9.13 & 7001 & 10.27 & 2527 & 8.89 \\
\hline $\begin{array}{l}\text { Officer/Warrant } \\
\text { Officer }\end{array}$ & 12600 & 9.95 & 6802 & 9.98 & 1804 & 6.35 \\
\hline \multicolumn{7}{|l|}{$\begin{array}{l}\text { Duration of service } \\
\text { (years) }\end{array}$} \\
\hline$<1$ & 58937 & 46.55 & 27764 & 40.75 & 12454 & 43.81 \\
\hline $1-2$ & 44189 & 34.90 & 25661 & 37.66 & 11248 & 39.57 \\
\hline $3-5$ & 12541 & 9.91 & 7659 & 11.24 & 2594 & 9.12 \\
\hline $5-30$ & 10936 & 9.64 & 7056 & 10.36 & 2133 & 7.50 \\
\hline
\end{tabular}

2-3 times higher in the pre-brace compared with the brace period. Rates were similar between the brace and post-brace periods except for vertebral column injuries, which were more common during post-brace (unadjusted OR 3.20, 95\% CI 1.11 to 9.21). However, the absolute number of vertebral column injuries was very small $(n=6$ brace and $n=8$ postbrace), making the estimated risk unstable.

Compared to the brace period, the adjusted OR for ankle injury was higher in the pre-brace and post-brace periods (table 4). Results were similar for severe ankle injuries, although the pre-brace period risk estimate was somewhat attenuated. When cases were restricted to those occurring during jump week, the magnitude of the adjusted OR for ankle injury was increased for both the pre-brace (OR 3.11,
95\% CI 2.41 to 4.03 ) and post-brace (OR $2.13,95 \%$ CI 1.51 to 3.02) periods (not shown).

After adjustment, ORs for other study outcomes were also about two during the pre-brace compared with the brace period. No statistically significant differences in non-ankle injury risks were observed between the brace and post-brace periods, but there was evidence of higher risk of multiple injuries in the post-brace compared with the brace period (table 4). The higher rate of multiple injuries in the postbrace period persisted when we redefined multiple injuries to exclude ankle diagnoses (not shown).

Cause of injury codes were recorded only for traumatic injury diagnoses. If some parachuting injuries had been diagnosed as musculoskeletal conditions rather than traumas

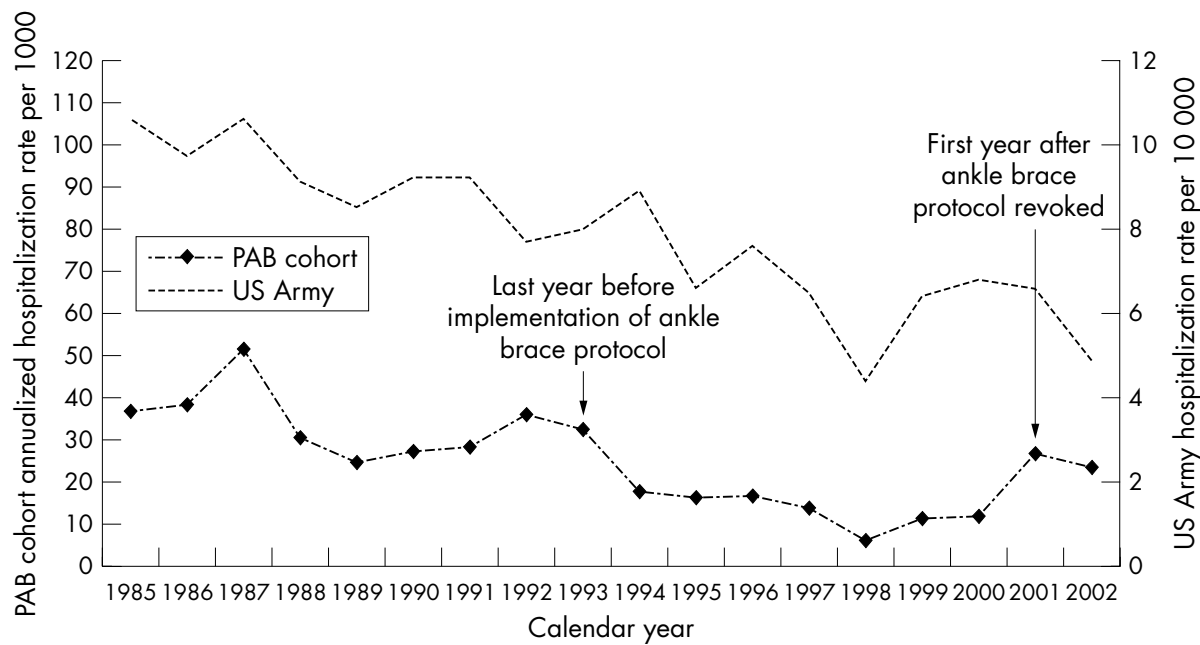

Figure 1 Annualized ankle injury hospitalization rates for $\mathrm{PAB}$ cohort (per 1000)* and for US Army cohort (per 10000 ). * Standardized to 1985 PAB population distribution (age, sex, and race). Note: US Army rates not standardized. Therefore, trends in US Army rates may be influenced by demographic changes. 
Table 3 Number ( $\mathrm{n}$ ) and percent (\%) of parachuting related* traumatic injuries requiring hospitalization $\dagger$ with unadjusted odds ratios (OR) and 95\% confidence intervals (CI) among US Army Airborne School trainees, by parachute ankle brace protocol, 1985-2002

\begin{tabular}{|c|c|c|c|c|c|c|c|c|c|c|c|}
\hline \multirow[b]{2}{*}{ Injury type } & \multicolumn{4}{|c|}{ Pre-brace protocol $\ddagger(n=126603$ ) } & \multicolumn{3}{|c|}{ Brace protocol $\ddagger$ ( $n=68 \quad 140)$} & \multicolumn{4}{|c|}{ Post-brace protocol $\ddagger(n=28$ 429) } \\
\hline & $\mathbf{n}$ & $\%$ & OR & $95 \% \mathrm{Cl}$ & $\mathbf{n}$ & $\%$ & OR & $\mathbf{n}$ & $\%$ & OR & $95 \% \mathrm{Cl}$ \\
\hline Ankle injury & 423 & 0.33 & 2.21 & $1.79-2.75$ & 103 & 0.15 & 1.00 & 71 & 0.25 & 1.65 & $1.22-2.24$ \\
\hline Severe ankle injury§ & 342 & 0.27 & 1.92 & $1.53-2.41$ & 96 & 0.14 & $1.00^{\circ}$ & 68 & 0.24 & 1.70 & $1.25-2.32$ \\
\hline $\begin{array}{l}\text { Musculoskeletal } \\
\text { injury }\end{array}$ & 136 & 0.11 & 1.63 & $1.16-2.28$ & 45 & 0.07 & $1.00^{\bullet}$ & 17 & 0.06 & 0.91 & $0.52-1.58$ \\
\hline $\begin{array}{l}\text { Any traumatic } \\
\text { (non-ankle) injury }\end{array}$ & 265 & 0.21 & 1.72 & $1.34-2.20$ & 83 & 0.12 & 1.00 & 32 & 0.11 & 0.92 & $0.61-1.39$ \\
\hline $\begin{array}{l}\text { Injury to multiple } \\
\text { body parts }\end{array}$ & 48 & 0.04 & 2.15 & $1.14-4.05$ & 12 & 0.02 & 1.00 & 9 & 0.03 & 1.80 & $0.76-4.27$ \\
\hline \multicolumn{12}{|c|}{$\begin{array}{l}\text { *Parachuting cause of injury code. } \\
\text { †Hospitalization occurring during a five week period from the start of jump week through the } 4 \text { th week following airborne training. } \\
\text { †ump week training dates were as follows: pre-brace }(01 / 01 / 85-30 / 09 / 93), \text { brace protocol }(01 / 01 / 94-30 / 09 / 00) \text {, post-brace }(01 / 10 / 00-31 / 12 / 02) \text {. } \\
\text { §Ankle injury hospitalization with ankle procedure. } \\
\text { - Referent group. }\end{array}$} \\
\hline
\end{tabular}

(that is, internal derangement of knee and other joints, dorsopathy, etc), a cause code would not have been included in the hospital record. When condition codes were included in the primary case definition, three ankle cases and 41 musculoskeletal cases (predominantly knee and vertebral column) were added. However, adjusted results were largely the same as those from our primary analysis, with the exception of a small decrease in the adjusted odds of musculoskeletal injury in the post-brace versus brace period (OR $0.83,95 \%$ CI 0.49 to 1.39 ) and a modest increase in the odds of multiple injury in the pre-brace compared with the brace period (OR 2.84, 95\% CI 1.33 to 6.08 ). The OR for knee cases during the post-brace period was reduced to OR 0.40 (95\% CI 0.12 to 1.36 ).

Controlling for protocol period, injury risk varied with the demographic characteristics of students. Age at the start of training was the strongest predictor of all types of injury, especially among those aged 25 years and older. Compared with Whites, Black students had a slightly higher risk of ankle injury, and students of "Other" race/ethnicity had a
$50 \%$ higher risk of musculoskeletal injury. Odds of musculoskeletal injury and any traumatic injury were higher among female compared with male students (table 4).

\section{DISCUSSION}

Use of an outside-the-boot ankle brace reduced the incidence of ankle injury hospitalization among Army Airborne School students without increasing injuries to other parts of the body. Compared with pre- and post-brace time periods, ankle injuries were $40-55 \%$ less common during the brace protocol period. Rates of non-ankle injuries were consistently highest during the pre-brace period and generally similar in brace compared with post-brace periods. In addition to ankle injuries, students trained during the post-brace period had higher, but not statistically significant, risk of multiple injuries and a statistically significant higher risk of vertebral column injury compared with those who trained during the brace period. Overall, patterns of injury across categories of age and sex were similar to those found in previous studies. $^{311}$

Table 4 Multivariate models for the prediction of various parachuting related* injury outcomes among US Army Airborne School trainees, 1985-2002

\begin{tabular}{|c|c|c|c|c|c|c|c|c|c|c|}
\hline \multirow[b]{2}{*}{ Model outcome } & \multicolumn{2}{|c|}{ Ankle injury } & \multicolumn{2}{|c|}{ Severe ankle injury† } & \multicolumn{2}{|c|}{ Musculoskeletal injury } & \multicolumn{2}{|c|}{ Any traumatic injury $\ddagger$} & \multicolumn{2}{|c|}{ Multiple injury } \\
\hline & OR§ & $95 \% \mathrm{Cl}$ & OR & $95 \% \mathrm{Cl}$ & OR & $95 \% \mathrm{Cl}$ & OR & $95 \% \mathrm{Cl}$ & OR & $95 \% \mathrm{Cl}$ \\
\hline \multicolumn{11}{|l|}{ Protocol } \\
\hline Pre-brace & 2.38 & $1.92-2.95$ & 2.05 & $1.63-2.57$ & 1.78 & $1.27-2.50$ & 1.81 & $1.42-2.32$ & 2.40 & $1.27-4.53$ \\
\hline Brace protocol & $1.00^{* *}$ & - & $1.00^{* *}$ & - & $1.00^{* *}$ & - & $1.00 * *$ & - & $1.00^{* *}$ & - \\
\hline Post-brace & 1.72 & $1.27-2.32$ & 1.77 & $1.30-2.42$ & 0.92 & $0.53-1.60$ & 0.96 & $0.64-1.44$ & 1.81 & $0.76-4.30$ \\
\hline \multicolumn{11}{|c|}{ Age at start of training } \\
\hline $17-18$ & $1.00^{* *}$ & - & $1.00^{* *}$ & - & $1.00 * *$ & - & $1.00 * *$ & - & $1.00^{* *}$ & - \\
\hline $19-20$ & 1.20 & $0.90-1.60$ & 1.36 & $0.99-1.87$ & 1.11 & $0.71-1.74$ & 1.18 & $0.86-1.63$ & 1.26 & $0.54-2.95$ \\
\hline $21-22$ & 1.79 & $1.33-2.42$ & 1.88 & $1.35-2.63$ & 1.22 & $0.74-2.02$ & 1.17 & $0.81-1.68$ & 1.46 & $0.57-3.70$ \\
\hline $23-24$ & 1.84 & $1.33-2.54$ & 2.01 & $1.41-2.87$ & 1.22 & $0.70-2.11$ & 1.31 & $0.88-1.93$ & 1.70 & $0.64-4.53$ \\
\hline $25-29$ & 2.71 & $2.03-3.63$ & 2.99 & $2.16-4.12$ & 1.63 & $0.99-2.68$ & 1.80 & $1.27-2.56$ & 2.77 & $1.17-6.56$ \\
\hline $30-34$ & 3.18 & $2.22-4.56$ & 3.42 & $2.30-5.10$ & 2.97 & $1.68-5.26$ & 2.38 & $1.53-3.69$ & 5.39 & $2.11-13.77$ \\
\hline $35-56$ & 4.89 & $3.04-7.85$ & 5.54 & $3.32-9.25$ & 4.07 & $1.86-8.91$ & 3.21 & $1.72-5.99$ & 3.67 & $0.78-17.36$ \\
\hline \multicolumn{11}{|l|}{ Sex } \\
\hline Men & & & & & $1.00 * *$ & - & $1.00^{* *}$ & - & & \\
\hline Women & & & & & 1.97 & $1.24-3.14$ & 1.74 & $1.23-2.47$ & & \\
\hline \multicolumn{11}{|l|}{ Race/ethnicity } \\
\hline White & $1.00^{* *}$ & - & & & $1.00 * *$ & - & & & $1.00^{* *}$ & - \\
\hline Black & 1.23 & $1.00-1.51$ & & & 0.67 & $0.42-1.05$ & & & 1.04 & $0.53-2.02$ \\
\hline Other & 1.25 & $0.97-1.60$ & & & 1.54 & $1.05-2.27$ & & & 1.95 & $1.04-3.63$ \\
\hline $\begin{array}{l}\text { *Parachuting caus } \\
\text { †Ankle injury hosp } \\
\text { †Excludes ankle in } \\
\text { §Odds ratio, adju } \\
\text { - Jump week train } \\
\text { **Referent categor }\end{array}$ & $\begin{array}{l}\text { jury co } \\
\text { ation w } \\
\text { all } m \\
\text { tes wer }\end{array}$ & $\begin{array}{l}\text { nkle proced } \\
\text { covariates. } \\
\text { follows: pre }\end{array}$ & ace 10 & $1 / 85-30 / 0$ & 3), brc & rotocol $(01 /$ & $194-30$ & 9/00), post- & $101 / 1$ & $0-31 / 12 / 02)$ \\
\hline
\end{tabular}


The results of this evaluation are in general agreement with two earlier studies that examined the effectiveness of the $\mathrm{PAB}$. In a one month randomized field trial, the rate of ankle injury among Airborne School trainees assigned to brace wear was $50 \%$ lower than the rate among non-brace wearers. ${ }^{6}$ This difference was largely driven by the higher rate of inversion ankle sprains among non-braced (3.79 per 1000 jumps) versus braced (0.55 per 1000 jumps) students. Other injuries occurred at similar rates for the two groups, with $5.3 \%$ of the non-braced and $4.6 \%$ of the braced group having at least one non-ankle injury.

Similar protective effects of PAB use were observed in a US Army Airborne Ranger battalion over a 38 month period from 1994-97. ${ }^{5}$ Specifically, the rate of ankle injury decreased from 4.5 per 1000 jumps to 1.5 per 1000 jumps after initiating mandatory $\mathrm{PAB}$ wear. No statistically significant differences in the rate of injury to other body parts were observed.

There are several limitations to this study to be considered when evaluating the results. First, since injury outcomes were ascertained from hospital records, changes in admission policies over time could strongly influence results. We calculated the rate of ankle injury hospitalization for all active duty US Army personnel, and found a 54\% decrease in admissions (from 10.6/10 000 to 4.9/10 000) between 1985 and 2002 (fig 1). If US Army-wide injury hospitalization rates stabilized after the late 1990s, comparisons between the brace and post-brace periods would provide the most valid estimates of the risks and benefits associated with PAB use. However, if injury hospitalization rates continued to decline over the entire study period, the OR for ankle injury in the post-brace period may underestimate the effectiveness of the $\mathrm{PAB}$ in reducing ankle injury.

Less severe injuries not requiring hospitalization-for example, most ankle sprains-were excluded from the case definition. However, evidence from the field trial, in which injuries were ascertained immediately after ground contact, supports the effectiveness of the PAB in also reducing ankle sprains. ${ }^{6}$ The current study cannot address the possibility that $\mathrm{PAB}$ use affects the risk of minor injuries, such as lower leg contusions, which are unlikely to result in hospitalization.

An additional limitation is the inclusion of trainees who left training before jump week. Although data concerning graduation status and the reason for non-graduation were obtained, information was not available regarding the timing of termination for the $15.7 \%$ of students who did not complete training. Failure to exclude these students would result in an overestimation of the number at risk and, hence, an underestimation of injury rates. However, since the proportion of non-graduates was similar for all brace protocol periods ( $16.2 \%$ pre-brace, $15.5 \%$ brace, $14.0 \%$ post-brace), any bias would be non-differential, and therefore the effect on the estimated association between PAB protocol period and injury risk should be minimal.

Finally, the use of a risk period that extends past the dates of training may have resulted in the inclusion of some nontraining injuries, but the number is likely to be small. Odds ratios were similar when cases were restricted to those occurring during jump week. The reduced precision resulting from the smaller number of cases, and the possibility of missing cases due to delayed treatment, led us to use the longer risk interval for these analyses.

Although subject to the above noted limitations, this study also has several strengths. Most noteworthy was the availability of an occupational cohort with good quality demographic and outcome data. If any students were missing from the roster data, the internal validity of the study would not be affected. Furthermore, the 18 year study interval, including two non-brace protocol periods, allowed evaluation of time trends in injury rates. The large size of the cohort also

\section{Key points}

- Between 1985 and 2002, ankle injuries accounted for $64 \%$ of parachuting-related hospitalizations experienced by US Army paratrooper trainees during the month beginning with the first static line jump.

- The odds of experiencing an ankle injury were twice as high when training occurred during intervals when the use of an outside-the-boot ankle brace was not required.

- The occurrence of other types of traumatic injuries was not increased during ankle brace use periods.

- The ratio of expenditures ( $\$ 30000$ per year) for braces to hospital care and rehabilitation costs saved (\$835000 per year) is 1:29.

permitted analysis of various injury outcomes and many covariates, although only a few were important predictors of injury.

Assuming a one year lifespan and a cost of $\$ 60$ per brace, the estimated total annual cost of the PAB is approximately $\$ 30000$ for purchase and replacement of 500 units. Using standard US Army methods to assess health hazards, resuming universal $\mathrm{PAB}$ use at the Airborne School could be expected to result in an annual cost avoidance of $\$ 865000 .^{12}$ These estimates capture only the costs associated with relatively severe injury requiring hospitalization, and none of the costs due to less severe injury. Therefore, the true cost avoidance associated with PAB use is likely to be higher. In addition, PAB use in settings outside the Airborne School (for example, during training exercises, proficiency jumps, and combat jumps) were not considered in the cost analyses, and may result in additional savings and improvements in military readiness.

In summary, the use of a $\mathrm{PAB}$ during airborne training appears to reduce the risk of ankle injury without increasing the risk of other types of traumatic injury. Assuming other training and hospital admission practices remain stable, approximately $40 \%$ of ankle injury hospitalizations currently experienced by US Army Airborne School trainees could be prevented if $\mathrm{PAB}$ use were reinstituted. As about $11 \%$ of ankle fractures among all US Army personnel in 2002 occurred during Airborne School training (not shown), use of the PAB could be expected to reduce the overall rate of ankle fracture in the Army by $4-5 \%$. Furthermore, reinstituting mandatory use of the PAB during training could potentially save $\$ 835000$ in hospitalization, disability, and outpatient care costs annually for trainees. Although the effectiveness of the PAB may vary depending on such factors as weather, parachute type, combat load, and terrain in the landing area, ${ }^{11}$ the PAB is likely to impart some measure of protection against ankle injury under most circumstances. Therefore, adoption of $\mathrm{PAB}$ use by non-training airborne units should be considered.

\section{Authors' affiliations \\ M D Schmidt, S I Sulsky, ENVIRON International Corp, Amherst MA, USA \\ P J Amoroso, US Army Research Institute of Environmental Medicine, Natick MA, USA}

\section{REFERENCES}

1 Lowdon IM, Wetherill MH. Parachuting injuries during training descents. Injury 1989;20:257-8. 
2 Farrow GB. Military static line parachute injuries. Aust N Z J Surg 1992;62:209-14

3 Craig SC, Morgan J. Parachuting injury surveillance, Fort Bragg, North Carolina, May 1993 to December 1994. Mil Med 1997;162:162-4.

4 Bricknell MC, Amoroso PJ, Yore MM. What is the risk associated with being a qualified military parachutist? Occup Med (Lond) 1999;49:139-45.

5 Schumacher JT Jr, Creedon JF, Pope RW. The effectiveness of the parachutist ankle brace in reducing ankle injuries in an airborne ranger battalion. Mil Med 2000; 165:944-8.

6 Amoroso PJ, Ryan JB, Bickley B, et al. Braced for impact: reducing military paratroopers' ankle sprains using outside-the-boot braces. J Trauma 1998:45:575-80.
7 Ekeland A. Injuries in military parachuting: a prospective study of 4499 jumps. Injury 1997;28:219-22.

8 Kragh JF Jr, Taylor DC. Parachuting injuries: a medical analysis of an airborne operation. Mil Med 1996;161:67-9.

9 Amoroso PJ, Yore MM, Weyandt B, et al. Chapter 8. Total Army injury and health outcomes database: a model comprehensive research database. Mil Med 1999;164(Suppl 8): 1-36.

10 Barrell V, Aharonson-Daniel L, Fingerhut LA, et al. An introduction to the Barell body region by nature of injury diagnosis matrix. Inj Prev 2002:8:91-6.

11 Knapik JJ, Craig SC, Hauret KG et al. Risk factors for injuries during military parachuting. Aviat Space Environ Med 2003;74:768-74.

12 Brett G, Evenden J, Spencer C. Health Hazard Assessment Database. In. 3. $1 \mathrm{~b}$ ed. McLean, VA: Logistics Management Institute, 1997.

\section{LACUNAE}

\section{New report on injury inequalities}

C

hildren from poor families are five times more likely to die from unintentional injuries, a new report from England's Health Development Agency highlights. The alarming fact that children from the poorest families are five times more likely to be killed as a result of unintentional injuries than those from the most affluent is one of the most stark and damning statistics to be highlighted by the report. Children from poorer families are also 16 times more likely to die in a house fire and 5 times more likely to die as a pedestrian. Research shows that the poorest children are more likely to suffer injuries that require hospital admission and when they are admitted their injuries are likely to be more serious than those experienced by children from affluent families. The report, Injuries in children aged 0-14 years and inequalities, looks at the variations in place, social, economic, and cultural factors, and how they affect the rate, severity, and nature of unintentional injuries that occur. The report, by Professor Elizabeth Towner, highlights many of the inequalities, points out the lack of appropriate interventions, and suggests how best these differences might be evened out and how threats posed to children might be minimised. The good news is that the number of unintentional injury deaths has been declining steadily. In England and Wales in 1979, almost 1100 children died as a result of unintentional injury, compared with 261 in 2002. The report can be accessed via http://www.hda.nhs.uk/documents/injuries in_children_inequalities.pdf. The report was prepared by the Health Development Agency Evidence and Guidance Collaborating Centre for the Prevention of Accidental Injuriesa consortium of organisations headed by Elizabeth Towner, Professor of Child Health at the University of the West of England. The other parts of the consortium are the University of Newcastle and the Child Accident Prevention Trust. 